\title{
Analysis of vibrations for detecting cracks in gears
}

\author{
A. Belsak \& J. Flasker \\ University of Maribor, Faculty of Mechanical Engineering, Slovenia
}

\begin{abstract}
A crack in the tooth root, which often results in failure of gear unit operation, is the least desirable among damages and faults, which can cause problems in gear unit operation. A fatigue crack in the tooth root brings about important changes in tooth stiffness, whereas other faults are usually associated with changes in other dynamic parameters. In this paper, fault analyses relating to gear units with real damages or faults are presented. Possible damage can be determined by monitoring vibrations. Time signals acquired by experiments are analysed by means of various methods. The Wavelets and Joint Time Frequency Analyses are used to analyse non-stationary signals.

Keywords: gear, failure, vibration, signal analysis.
\end{abstract}

\section{Introduction}

The aim of maintenance is to keep a technical system (e.g. a gear-unit) in the most suitable working condition; its purpose is to discover, to diagnose, to foresee, to prevent and to eliminate damages. The purpose of diagnostics is to define the current condition of the system, and the location, shape and reason of the damage formation. A gear unit has elements that make transmission of rotating movement possible, and as such, it is a complex dynamic model. In spite of this, its movement is usually periodical, and faults and damages represent a disturbing quantity or impulse, which is indicated by local and time changes in vibration signals $[1,2]$. As a result, time-frequency changes can be expected [3]. This idea is based on kinematics and operating characteristics.

It is possible, by using time-frequency analysis, to describe in what way frequency components of non-stationary signals change with time as well as to define their intensity. Adaptive and wavelet transforms are representatives of 
various time-frequency algorithms [4]. The basic idea of all linear transforms is to perform a comparison with elementary functions determined in advance [5]. By means of different elementary functions, various signal presentations can be acquired [6].

\section{Adaptive method analysis}

Many authors had been developing algorithms with no interferences, reducing the usability of individual transforms as opposed to Cohen's class, but the adaptive transform of a signal was significantly improved by Qian and Chen [7]. Adaptive transform of a signal $x(t)$ is expressed as follows:

$$
x(t)=\sum_{p} B_{p} \cdot h_{p}(t)
$$

The following equations are used to define analysis:

$$
B_{p}=\left\langle x, h_{p}\right\rangle
$$

Thereby similarity between the measured signal $x(t)$ and elementary functions $h_{p}(t)$ of transform is expressed. It is possible to define time-dependent adaptive spectrum in the following way:

$$
P_{A D T}(t, \omega)=\sum_{p}\left|B_{p}\right|^{2} \cdot P_{W V} h_{p}(t, \omega)
$$

This is an adaptive spectrogram that is based on representations; it has no interferences and no cross terms. This is what it makes it different from the Wigner-Ville distribution. Besides it also meets the requirements in concern to energy conservation.

When it comes to linear presentations, it is very important to select the relevant elementary functions. In relation to adaptive representation, elementary functions are fairly demanding. The adaptive transform generally does not depend on the selected elementary functions as it allows arbitrary elementary functions. Elementary functions, used for adaptive representation of a signal with Eq. (1), are very general in theory but not always so in practice. When it comes to time and frequency, elementary functions are preferentially localised so as to emphasize the time dependence of a signal.

\section{Wavelet analysis}

The continuous wavelet transform of function $x(t) \in L^{2}(\Re)$ at the time and scale is expressed as follows [8]: 


$$
\begin{aligned}
W x(u, s)=\left\langle x, \psi_{u, s}\right\rangle & =\int_{-\infty}^{+\infty} x(t) \cdot \frac{1}{\sqrt{s}} \cdot \psi^{*}\left(\frac{t-u}{s}\right) \cdot d t=x(t) \otimes \bar{\psi}_{s}(t) \\
& \bar{\psi}_{s}(t)=\frac{1}{\sqrt{s}} \cdot \psi^{*}\left(\frac{-t}{s}\right) \\
& \bar{\Psi}_{s}(\omega)=\sqrt{s} \cdot \Psi^{*}(s \cdot \omega)
\end{aligned}
$$

where the transform is presented as the product of convolution; in Eq. (6), the expression of an average wavelet function and the corresponding Fourier integral transform, Eq. (7), are presented.

When it comes to the continuous wavelet transform, the observed function $x(t)$ is multiplied by a group of shifted and scaled wavelet functions. In this way, it comes to changes in time and frequency dissemination of the continuous wavelet transform.

Wavelets are locally limited functions. As such they are applied to analyse the observed function $x(t)$. The continuous wavelet transform is very sensitive to local non-stationarities. Gabor wavelet function is a representative of an approximately analytical wavelet function, acquired by means of a frequency modulation of the Gauss window function [8]. A family of wavelet functions, or shifted and scaled Gabor wavelet function is acquired in the following way (8):

$$
\psi_{\text {Gabor }}(t, \sigma, \eta)=\frac{1}{\sqrt{s}} \cdot \frac{1}{\sqrt[4]{\sigma^{2} \cdot \pi}} \cdot e^{-\frac{1}{2 \cdot \sigma^{2}} \cdot\left(\frac{t-u}{s}\right)} \cdot e^{i \cdot \eta \cdot\left(\frac{t-u}{s}\right)}
$$

\section{Practical analysis}

The test plant (Fig. 1) of the Faculty of Mechanical Engineering, University of Maribor was used to carry out the measurements. A one-stage helical gear unit is located at the spot where vibrations were measured.

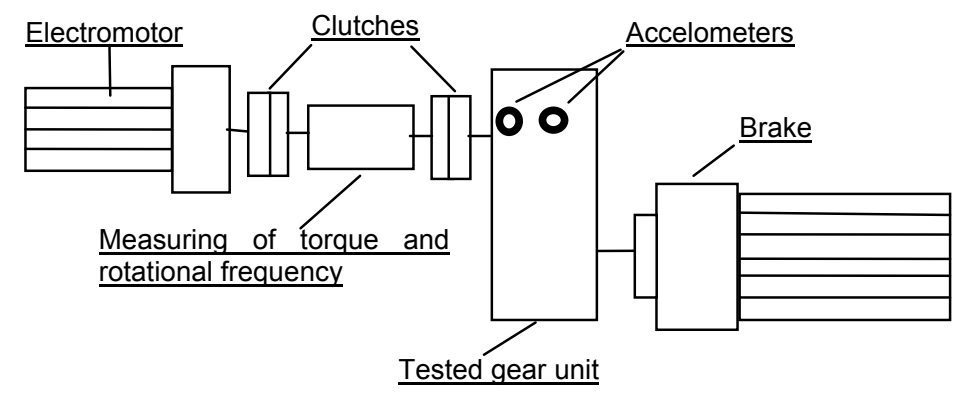

Figure 1: Test plant. 
Each gear unit had a carburised spur gear pair of module $4 \mathrm{~mm}$. The pinion had 19, whereas the wheel had 34 teeth. A nominal pinion torque was $20 \mathrm{Nm}$ and nominal pinion speed was $1200 \mathrm{rpm}(20 \mathrm{~Hz})$. Tests were carried out under constant loads. The operating conditions were typical of this type of a gear unit. Accelerometers were fixed on the housings to perform the measurements.

A ground gear pair was a standard gear pair, but it had a crack in the tooth root of a pinion. It is shown in Fig. 2. The length of the crack on one of the teeth shown in Fig. 2 is $4.8 \mathrm{~mm}$. The whole measurement process and analysis preparations are presented in [9].

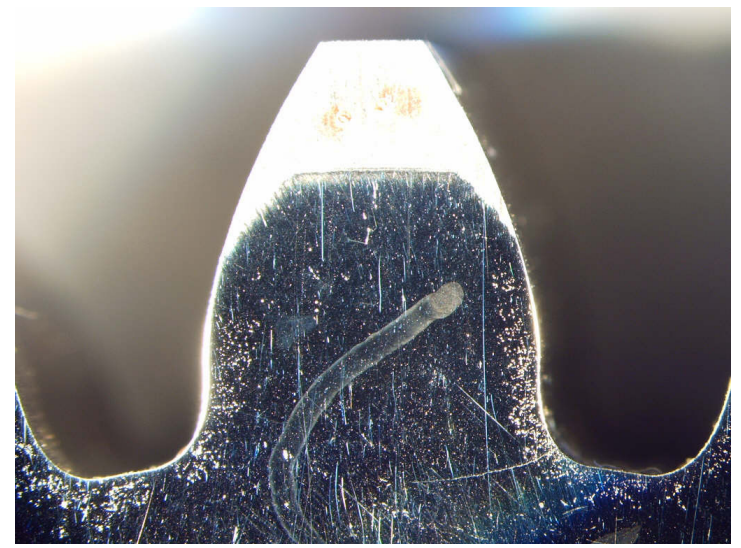

Figure 2: Pinion with a crack in the tooth root.
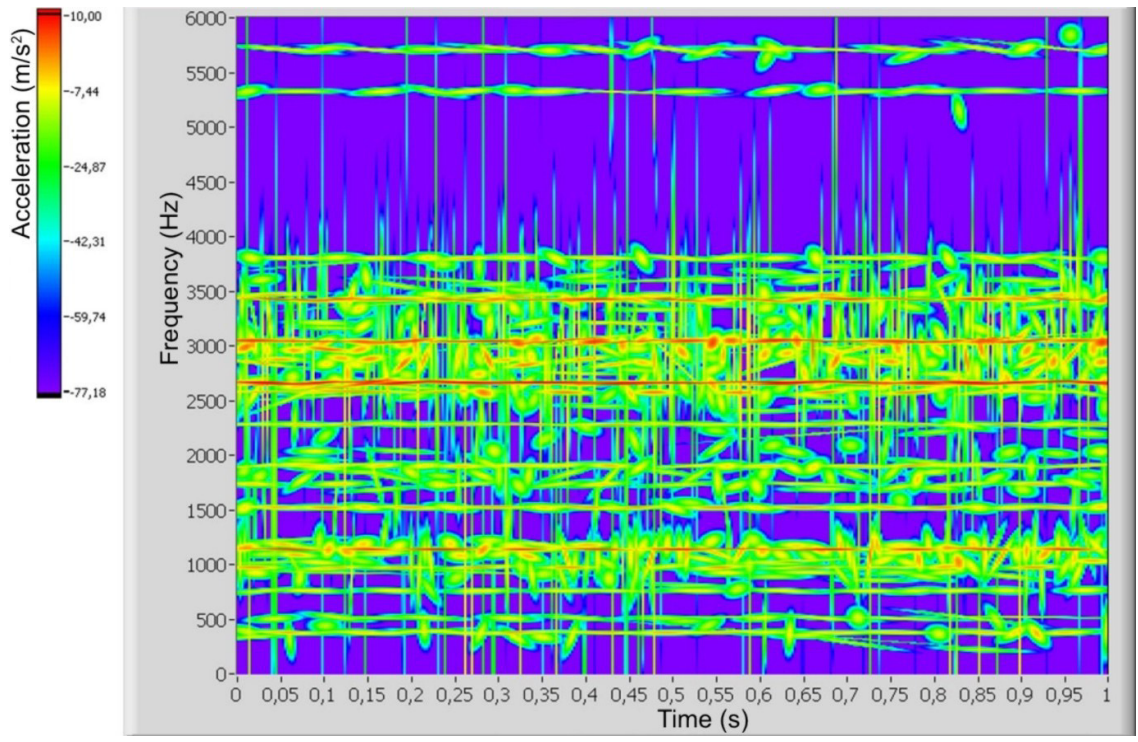

Figure 3: Adaptive spectrogram of a faultless gear unit. 
The signal of measured values is $1 \mathrm{~s}$ long and composed of, on an average, 12500 measuring points. In adaptive spectrogram (Fig. 3), no rhythmic pulsation of harmonics is evident, with the exception of typical frequencies. Some pulsation sources with higher level of energy accumulation in the origins can be noted. It is interesting to observe how frequency components with rotational frequency of $20 \mathrm{~Hz}$ increase or decrease (complete disappearance). This can be observed in relation to the 3 rd harmonic of mesh frequency. The value of $1530 \mathrm{~Hz}$ is expressed only in relation to the presence of a crack. This phenomenon is shown in Fig. 4, pulsation (i.e. the area, marked with a continuous line) is expressed, reflecting a single engagement of a gear pair with a crack within one rotation of a shaft. Also, between the 6th and the 9th harmonics (i.e. the area marked with a dashed line), sources indicating pulsating portions of individual components with the frequency of $20 \mathrm{~Hz}$ can be noted.

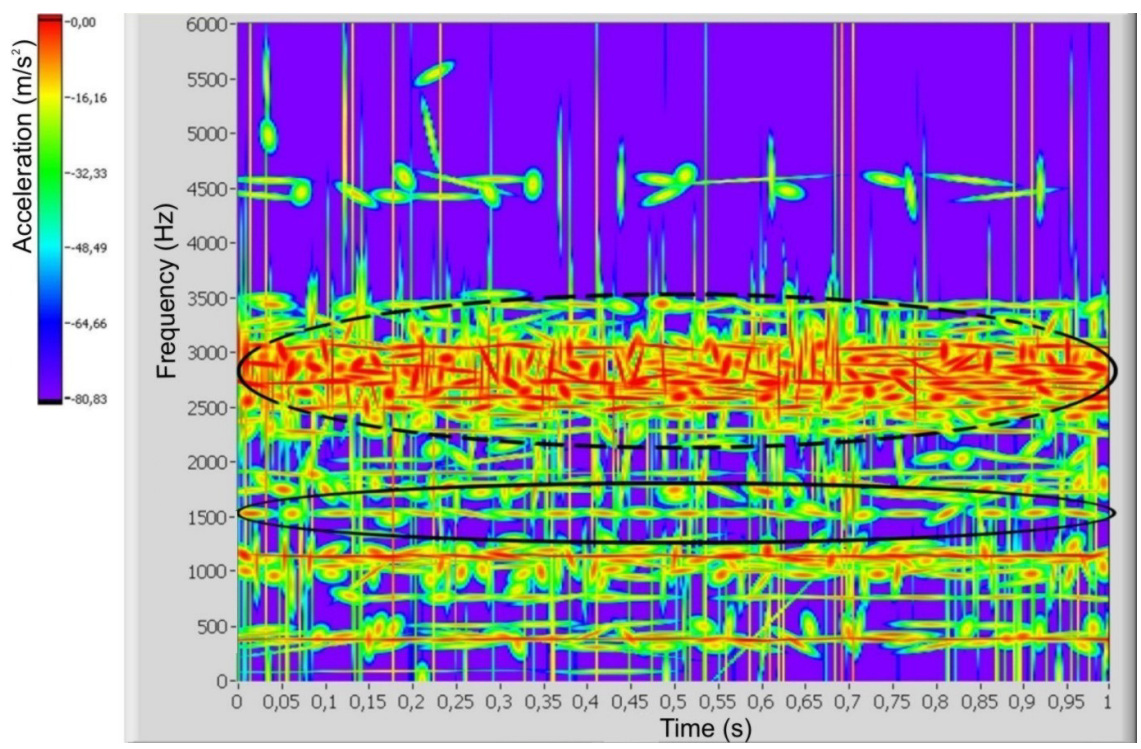

Figure 4: $\quad$ Adaptive spectrogram of a gear unit with a pinion with a crack.

A scalogram of analytical wavelet transform with Gabor wavelet function represents normalised and square values of amplitudes of wavelet coefficients. The connection between the scale and frequency is made, the representation is carried out in a time-frequency domain. This is very suitable for technical diagnostics because it is much simpler to find adequate characteristics in timefrequency domain (frequency scalogram) than in time-scale domain (scalogram). The goals of the analysis are to define the condition relating to the presence of a crack in a tooth root and to identify the location of the crack, i.e. the appropriate tooth. The continuous wavelet transform, parameters $\eta=6$ and $\sigma=1$, is used for 
574 High Performance Structures and Materials V
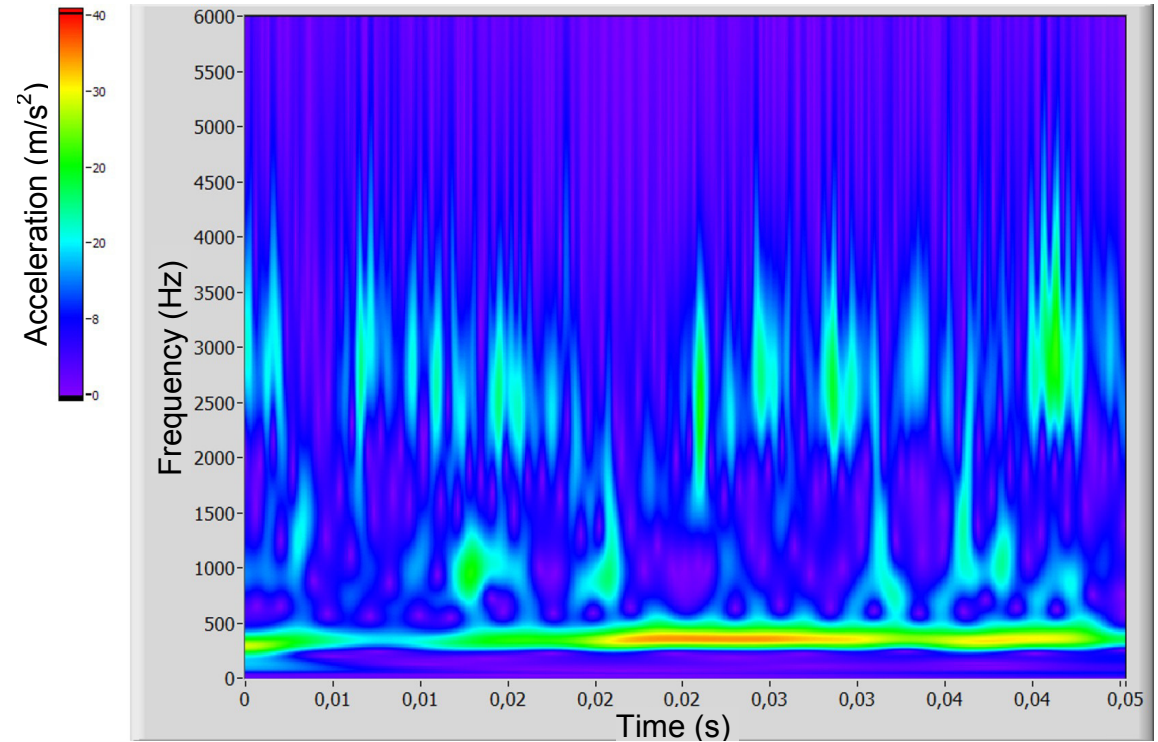

Figure 5: Average gabor frequency scalogram of square wavelet coefficient of the reference gear unit.

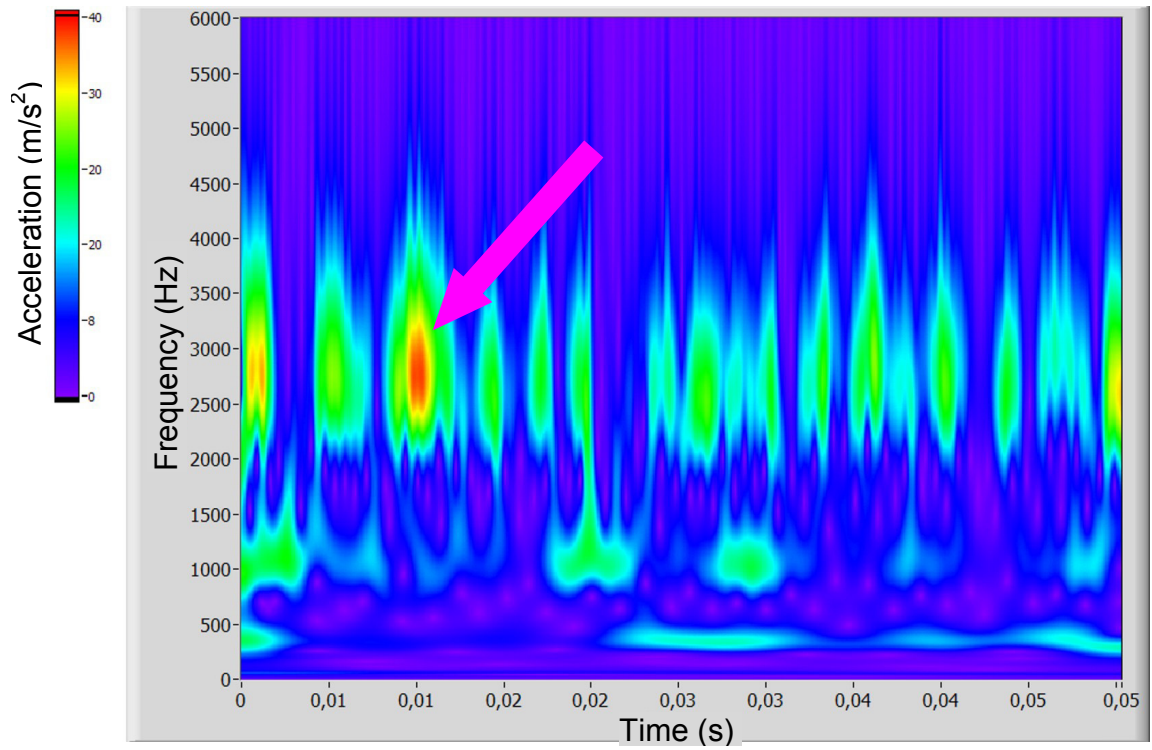

Figure 6: Average gabor frequency scalogram of square wavelet coefficient of the gear unit with a gear with a crack in a tooth root. 
the analysis. A part of the signal, representing one whole rotation of the tooth, i.e. of a pinion with a crack, of $50 \mathrm{~ms}$, is taken into account. The figures in the frequency scalogram associated with the faultless gear indicate that no particularities in expressed components occur that would denote local changes, which applies for a square representation (Fig. 5) of wavelet coefficients. This is, however, quite different in relation to the signal produced by a gear with a crack: a local change in wavelet coefficients can be noted, in time, at the value of $11 \mathrm{~ms}$, in frequency scalograms (Fig. 6). It is possible to observe local changes, i.e. the presence of transients, where there is the tooth with the crack in its root. The wavelet length is $50 \mathrm{~ms}$, which represents one rotation of the pinion, and there are 19 teeth along the circumference. The increased amplitude is located at $11 \mathrm{~ms}$ and belongs, therefore, to the fourth tooth in the direction of rotation from the reference positional point of the gear unit.

\section{Conclusion}

Vibration analysis aimed at identifying faults in industrial gear units is presented; on the basis of described methods, the safety of operation and, consequently, the reliability of monitoring operational capabilities can be improved.

By using appropriate Adaptive spectrogram samples and a clear presentation of the pulsation of individual frequency components it is possible to monitor the condition of a gear unit in a more reliable way; along with the average spectrum they form a criterion for evaluating the condition of a gear unit.

By using a wavelet transform changes cane be determined in a very short time, and the presence of damage can be identified at the level of an individual tooth. With an appropriate method or criterion, the actual condition of a gear unit and its vital component parts can be monitored, which can influence the operational capability significantly. In time detection of faults and damages significantly increases the reliability of operation control.

\section{References}

[1] Derrek, J. S., Gear Noise and Vibration, Marcel Dekker, New York, 1999.

[2] Taylor, J. I., The Vibration Analysis Handbook, Vibration Consultants, 1994.

[3] Mertins A., Signal Analysis, John Wiley \& Sons Inc., New York, 1999.

[4] Fladrin P., Time-Frequency/Time-Scale Analysis, Academic Press, San Diego, 1999

[5] Chen, V. C., Ling, H., Time-Frequency Transforms, Artech House Publishers, Boston, 2002

[6] Adewusi, S. in AI-Bedoor, B., Wavelet analysis of vibration signals of an overhang rotor with a propagating transverse crack, Journal of Sound and Vibration, 246(5), (2001) 777-793,

[7] Qian, S., Chen D., Joint Time-Frequency Analysis, Prentice Hall, Upper Saddle River, 1996 
576 High Performance Structures and Materials V

[8] Mallat, S., A Wavelet Tour of Signal Processing, Academic Press, San Diego, 1999

[9] Belsak, A., Time-Frequency Analysis of the Condition of Gear Units (Abstract in English), Doctoral Thesis, University of Maribor, Faculty of Mechanical Engineering, 2006. 